\title{
INSTRUMENTOS MAIS UTILIZADOS NA AVALIAÇÃO DA FUNCIONALIDADE DE IDOSOS COM DEMÊNCIA: UMA REVISÃO SISTEMÁTICA
}

\author{
MOST USED INSTRUMENTS IN THE ASSESSMENT OF FUNCTIONALITY IN THE ELDERLY WITH \\ DEMENTIA: A SYSTEMATIC REVIEW
}

\section{Mariana Luciano Almeidaa , Juliana Cerqueria Leite ${ }^{b}$, Aryanne Pereira Soares Avalhais ${ }^{c}$, Beatriz Rodrigues de Souza Melod, Catia Cristina Thomaze, Zilda Rezende Mota Botanf, Carlene Souza Silva Manzinis, Francisco Assis Carvalho Vale ${ }^{\mathrm{h}}$}

\author{
amarialmeida_18@yahoo.com.br, bjuliana_cleite@yahoo.com.br, caryannepsa@hotmail.com, dnursebia@hotmail.com, \\ ecatia.thomaz@hotmail.com, ${ }^{\mathrm{f}}$ zildaenfermeira@yahoo.com.br, ${ }^{\mathrm{g}} \mathrm{c}$ carlotamanzi@hotmail.com, ${ }^{\mathrm{h}}$ facvale@ufscar.br \\ Universidade Federal de São Carlos - São Carlos (SP), Brasil
}

Data de recebimento do artigo: 23/07/2015 Data de aceite do artigo: 15/10/2015

\section{RESUMO}

Introdução: Com o crescimento no número de idosos, a demência surge como uma das consequências das doenças crônico-degenerativas mais impactantes na funcionalidade de idosos. Objetivos: Descrever os instrumentos mais utilizados em estudos de avaliaçáo da funcionalidade de idosos com demência. Materiais e métodos: Realizou-se uma revisão sistemática de artigos que abordavam o presente assunto entre os anos de 2009 e 2014 nos idiomas português, inglês e espanhol. As bases de dados exploradas foram: SciELO, LILACS, Banco de Teses da CAPES, PubMed, Web of Science, PsycINFO e Scopus. Para o processo de identificação e seleção dos artigos encontrados nas bases de dados, utilizou-se o método PRISMA. Resultados: Foram identificados 62 instrumentos diferentes que avaliam a funcionalidade de idosos com demência, e nesse sentido houve a necessidade de destacar apenas os mais frequentes. $\mathrm{O}$ critério de inclusão nos resultados foi inserir apenas os instrumentos encontrados em mais de quatro estudos. Dentre os mais utilizados foram identificados seis instrumentos, os quais corroboram os encontrados por outras revisóes sistemáticas recentes. Conclusão: Encontrou-se que a Escala de Atividades Instrumentais de Vida Diária de Lawton e Brody, o Índice de Katz, o Questionário de Atividades Funcionais de Pfeffer, o DAD, o Índice de Barthel e o ADCS-ADL são os instrumentos mais utilizados na avaliação da funcionalidade de idosos com demência.

Palavras-chave: Avaliação; atividades cotidianas; demência; idoso.

\section{ABSTRACT}

Introduction: With the growth of the elderly population, dementia emerges as one of the most striking consequences of chronic degenerative diseases on the functionality of the elderly. Objective: To describe the instruments used by most studies for the evaluation of functionality in the elderly with dementia. Materials and methods: A systematic review was carried out of papers in Portuguese, English and Spanish that addressed this matter between the years 2009-2014 . The selected databases were: SciELO, LILACS, CAPES Theses Database, PubMed, Web of Science, PsycINFO and Scopus. For the process of identifying and selecting the items found in the databases, we used the PRISMA method. Results: 62 different instruments that evaluate functionality in elderly people with dementia were identified, and in that sense it was necessary to highlight only the most frequent. The criteria for inclusion in the results was to only introduce the instruments found in more than four studies. Six instruments were identified among the most used, which corroborate those found in other recent systematic reviews. Conclusion: We found that Lawton and Brody Instrumental Activities of Daily Living Scale, Katz Index, Pfeffer Functional Activities Questionnaire, DAD, Barthel Index and ADCS-ADL are the most used instruments in evaluation of the functionality in elderly patients with dementia.

Keywords: Assessment; daily activities; dementia; elderly. 


\section{INTRODUÇÃO}

Estudos enfatizam que a medida que o número de idosos cresce, a prevalência e a incidência de doenças crônico-degenerativas aumentam expressivamente e, entre essas, a demência se realça como agente importante de morbimortalidade, compondo o sexto grupo de doenças mais impactantes na funcionalidade e na mortalidade de idosos ${ }^{1,2}$. São comuns, na população idosa, queixas sobre o declínio da cognição e da dependência nas atividades funcionais, sendo forte a relação entre nível cognitivo e habilidade funcional ${ }^{3}$.

Segundo Gordilho ${ }^{4}$, capacidade funcional é a capacidade de manter as habilidades físicas e mentais necessárias a uma vida independente e autônoma. Sua prevalência geralmente é mensurada por meio da incapacidade em realizar as Atividades de Vida Diária (AVD), como as Atividades Básicas de Vida Diária (ABVD) e as Atividades Instrumentais de Vida Diária (AIVD). As $\mathrm{ABVD}$, descritas como atividades de autocuidado como tomar banho, pentear os cabelos, alimentar-se, usar o banheiro, são essenciais à sobrevivência diária, e as AIVD, ações envolvendo a organização da rotina diária como vestir-se, comer, preparar uma pequena refeição, caminhar dentro de casa ou pela vizinhança, fazer compras, emitir julgamentos, ler e escrever, discar ou atender telefone, são atividades necessárias para sustentar uma vida independente na comunidade 5 .

Para Matsudo ${ }^{6}$, capacidade funcional pode ser definida como o potencial que os idosos apresentam para decidir e atuar em suas vidas de forma independente no seu cotidiano, apesar de possuírem uma incapacidade física, mental ou social. Destacam-se atividades que são desenvolvidas diariamente e estão diretamente relacionadas ao autocuidado, ao cuidado de seu entorno e à participação social, constituindo, portanto, um fator importante para a saúde e o bem-estar dos idosos.

A funcionalidade, fundamental para o envelhecimento humano, trata das condições de saúde e da interação entre as capacidades física e psicocognitiva para a realização de atividades do cotidiano. $\mathrm{O}$ conceito em questão é influenciado pelo processo de envelhecimento fisiológico por características de gênero, idade, classe social, renda, escolaridade, condiçóes de saúde, cognição, ambiente, história de vida e por recursos de personalidade. Além disso, a funcionalidade representa um continuum de estados funcionais com vários graus possíveis de desempenho ou, olhando sob o aspecto negativo, vários graus de comprometimento ${ }^{7}$.

Nesse sentido, a funcionalidade é definida como um termo guarda-chuva para as funçóes e estruturas do corpo, atividades e participação. Diz respeito aos aspectos positivos da interação entre um indivíduo com a sua condição de saúde e seus fatores contextuais individuais (fatores ambientais e pessoais). A incapacidade denota os aspectos negativos dessa interação das disfunçóes nos sistemas orgânicos, seja nos aspectos fisiológicos ou morfológicos, seja na limitação das atividades, podendo resultar na restrição da participaçáo ${ }^{6}$.

Como o estado funcional é uma dimensão base para a avaliação gerontológica, muitos instrumentos específicos para determinação da avaliação das AVD são propostos e amplamente utilizados, com destaque para o Índice de Katz e para a Medida de Independência Funcional (MIF), que avaliam as ABVD, e para o Índice de Lawton-Brody e o Índice de Pfeffer, que avaliam as AIVD. Esses instrumentos, entretanto, ainda são utilizados de forma assistemática ${ }^{8}$ no Brasil.

Diante do exposto, pode-se observar que a funcionalidade é um conceito em construção que abarca diversas explicaçóes e pode ser avaliado sob diferentes aspectos. Visto que ela complementa a avaliação multidimensional de casos de demência, é interessante investigar quais instrumentos de avaliação da funcionalidade estão sendo utilizados em pesquisas que avaliam idosos com demência. Assim, este estudo objetiva descrever quais são os instrumentos mais utilizados pela literatura para a avaliação da funcionalidade de idosos com demência.

\section{MÉTODOS}

Realizou-se uma revisão sistemática dos últimos cinco anos (2009 a 2014) de artigos que utilizaram instrumentos de avaliação da funcionalidade em idosos com demência. As buscas foram realizadas no mês de maio de 2014 nas seguintes bases de dados: SciELO, LILACS, Banco de Teses da CAPES, PubMed, Web of Science, PsycINFO e Scopus. Para essas buscas foram utilizados os seguintes descritores: "assessment", "questionnaire", "aged", "activities of daily living" e "dementia" (indexados no MeSH e DeCS); "scale", "instrument", "elderly" e "functionality” (não indexados no MeSH e DeCS).

As estratégias utilizadas nas buscas foram elaboradas com base nos descritores apresentados acima, utilizando o operador booleano "AND" em todas as combinaçóes. Elas foram divididas em dois grupos de estratégia: o primeiro grupo corresponde à relação de "functionality" com os demais descritores; o segundo, à relaçáo de " $a c$ tivities of daily living" com os outros descritores, totalizando 16 estratégias de busca.

Para cada base de dados foram utilizados filtros com o objetivo de refinar e restringir a busca. Foram procurados artigos entre os anos 2009 e 2014 no idioma inglês, português e espanhol para todas as bases. $\mathrm{Na}$ SciELO, além desses filtros, utilizou-se restrição de áreas temáticas com a indicação das ciências da saúde. $\mathrm{Na}$ LILACS, a busca foi realizada por título e se optou por 
artigos com texto completo, além de se ter requisitado os artigos como tipo de documento. Os idosos foram indicados como sujeitos do estudo. Em relação ao PubMed, realizou-se a busca por titlelabstract, free full text e full text; além disso, foi indicado como aged pessoas com 65 anos ou mais, e journal article e classical article como article types. No Web of Science, a busca foi realizada por título, e as áreas de pesquisa selecionadas foram geriatrics gerontology, psychology, neurosciences neurology, psychiatry, rehabilitation e nursing. Além disso, o tipo de documento indicado foi o artigo. No PsycINFO utilizou-se any fields na busca, indicou-se pessoas com 65 anos ou mais como aged group e full text. No Scopus a busca foi realizada selecionando article title, abstract, keywords, artigos como document type, medicine, neuroscience, nursing, psychology e health professions como subjetc area e journals como source type. Em relação ao Banco de Teses da CAPES, não foi utilizado nenhum tipo de filtro nas buscas.

Além disso, é importante destacar que em um primeiro momento as buscas nas bases de dados foram realizadas por dois grupos de pesquisadores separadamente, sendo que em um segundo momento os pesquisadores entraram em um consenso em relaçáo aos resultados obtidos.

Para o processo de identificação e seleção dos artigos encontrados nas bases de dados, utilizou-se o método Preferred Reporting Items for Sistematic Review and Meta-Analyses (PRISMA) ${ }^{9}$, que tem o objetivo de auxiliar os autores a melhorar a comunicaçấo de revisóes sistemáticas e metanálises, além de poder ser utilizado para avaliaçôes críticas de revisões sistemáticas publicadas. $\mathrm{Na}$ etapa de análise e extração dos dados, utilizou-se uma adaptaçáo do instrumento para coleta de dados de artigos científicos proposto por Ursi ${ }^{10}$.

Desses 3125 artigos encontrados, 2050 estavam duplicados e foram removidos intra e interbases, restando 1075 para exclusão pela leitura de título e resumo dos artigos. Para o processo de exclusáo de artigos duplicados utilizou-se o programa Minilist.

Após essa etapa, foram excluídos 935 artigos que não tinham relação com instrumentos de avaliação da funcionalidade de idosos com demência. No total restaram 140 artigos nessa primeira fase de exclusão, os quais foram selecionados para a elegibilidade com leitura do texto na íntegra.

Como critério de inclusão, os 140 artigos selecionados deveriam apresentar em sua metodologia a utilização de algum instrumento com o objetivo de avaliar a funcionalidade de idosos com demência. Os artigos que não atingiram tal critério foram excluídos com justificativa.

Desses artigos selecionados para leitura na íntegra, 30 foram excluídos com as seguintes justificativas: os estudos não avaliavam idosos com demência, não especificavam o tipo de demência estudado, não utilizavam instrumentos de avaliação da funcionalidade, eram repetidos ou não se caracterizavam como artigo. Diante disso, 110 estudos foram incluídos para a análise e extração dos dados, conforme é apresentado pela Figura 1, baseada no método PRISMA.

Figura 1: Identificação e seleção dos artigos encontrados na presente revisão.
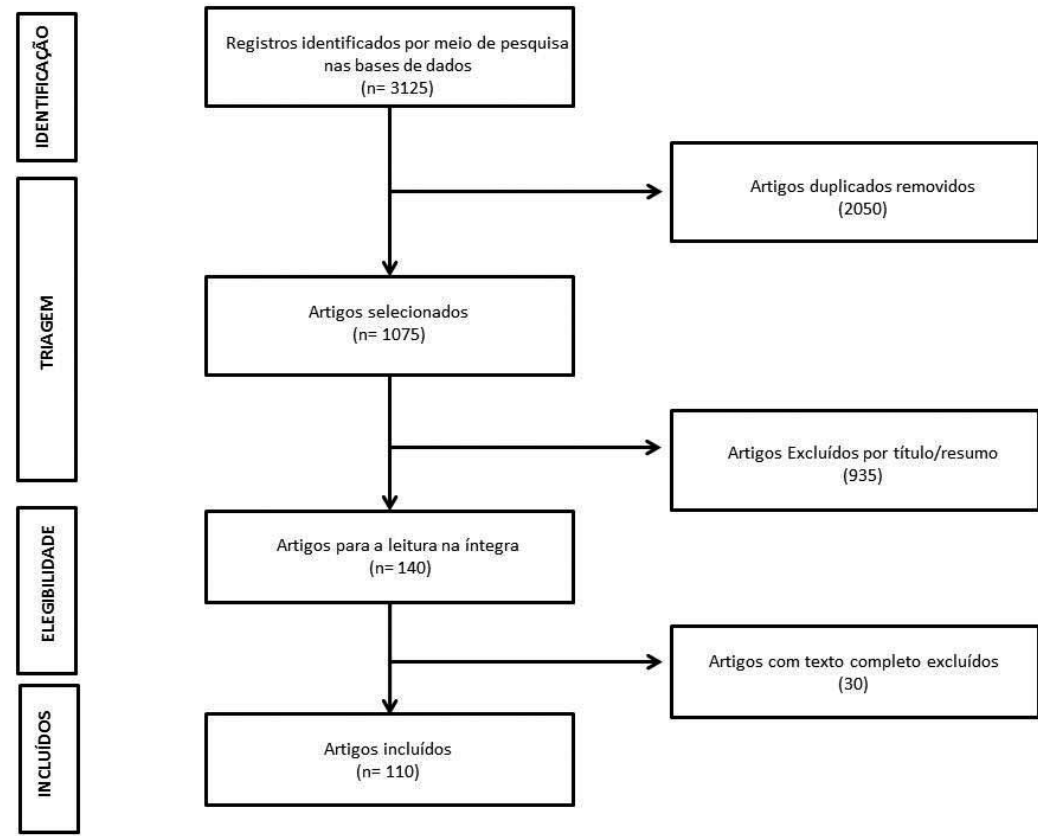

Rev. Aten. Saúde., São Caetano do Sul, v. 14, n. 48, p. 76-85, abr.jun., 2016 


\section{RESULTADOS}

Foram identificados 62 instrumentos diferentes que avaliam a funcionalidade de idosos com demência, portanto houve a necessidade de destacar apenas os mais frequentes. Como critério de inclusão nos resultados foram inseridos apenas os instrumentos encontrados em mais de quatro estudos. Dentre os mais utilizados foram identificados seis instrumentos; o número de estudos em que foram utilizados e os países nos quais foram produzidos estão apresentados na Tabela 1.

Tabela 1: Instrumentos utilizados com maior frequência no mundo, entre os anos de 2009 e 2014.

\begin{tabular}{|c|c|c|c|}
\hline Instrumento & Ano de elaboraçáo e primeiro autor & País & Tipo de Demência \\
\hline \multirow{6}{*}{ 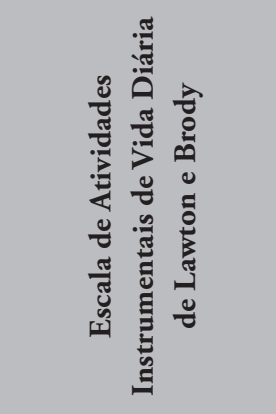 } & 2009. Fukui, T. & Japão & $\mathrm{DA}^{\mathrm{a}}$ \\
\hline & 2010. Marra, TA; Farina, E; Miyamoto, Y. & Brasil, Itália, Japão & $\begin{array}{l}\text { DA; DA, DV }{ }^{\mathrm{b}}, \mathrm{DFT}^{\mathrm{c}} \mathrm{e} \\
\text { DDP }^{\mathrm{d}} ; \mathrm{DA} \text { e DV }\end{array}$ \\
\hline & $\begin{array}{l}\text { 2011. Jonas, C; Rasovska, H.; Watmo, C; } \\
\text { Mougias, AA; Balci, BD. }\end{array}$ & $\begin{array}{c}\text { Alemanha, Alemanha, República Tcheca, } \\
\text { Suécia, EUA, Turquia }\end{array}$ & $\begin{array}{l}\text { DA; DDP e DA; DA; } \\
\text { DA; DA; }\end{array}$ \\
\hline & $\begin{array}{l}\text { 2012. Monaci, L; D’Onofrio, G; } \\
\text { Vreugdenhil, A. }\end{array}$ & Inglaterra, Itália, Austrália & DA; DA e DV; DA; \\
\hline & $\begin{array}{l}\text { 2013. Christ, JBM; Tran, M; Paula, JJ; } \\
\text { Hesseberg, K; Amanzio, M; Manni, R. }\end{array}$ & $\begin{array}{c}\text { Alemanha, Canadá, Brasil, Noruega, } \\
\text { Itália, Itália }\end{array}$ & $\begin{array}{c}\text { DDP; DA; DA; DA; } \\
\text { DA; DA }\end{array}$ \\
\hline & 2014. Koskas, P. & França & $\mathrm{DA}$ \\
\hline \multirow{4}{*}{ 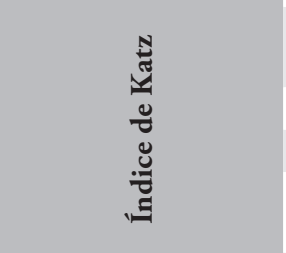 } & 2010. Marra, TA; Farina, E. & Brasil, Itália & $\begin{array}{l}\text { DA; DA, DV, DFT e } \\
\text { DDP. }\end{array}$ \\
\hline & 2011. Mougias, AA; Hebert, LE; Palmer, K. & Suécia, EUA, Itália & DA; DA;DA \\
\hline & 2012. D’Onofrio, G; Monaci, L. & Itália, Reino Unido & DA, DV; DA \\
\hline & $\begin{array}{l}\text { 2013. Paula, JJ; Lee, SB; Rolland, Y; } \\
\text { Amanzio, A. }\end{array}$ & Brasil, Coreia, França, Itália & $\begin{array}{l}\text { DA; DA, DV, DFT, } \\
\text { DL' }^{\mathrm{e}} \text { DDP; DA; DA }\end{array}$ \\
\hline \multirow{5}{*}{ 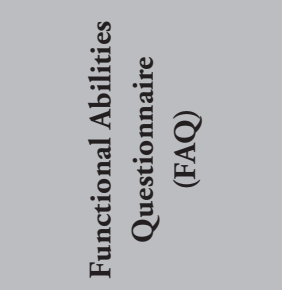 } & 2009. Diaz, CYE. & Cuba & DA; \\
\hline & 2010. Bahia, VS; Teng, E; Marra, TA. & Brasil, EUA, Brasil & DA; DA; DA \\
\hline & $\begin{array}{l}\text { 2011. Marshall, GA; Clare, L; Brown, PJ; } \\
\text { Gill, DP. }\end{array}$ & EUA, Reino Unido, EUA, EUA & $\begin{array}{l}\text { DA; DA; DA; DA e } \\
\text { DL }\end{array}$ \\
\hline & 2012. Nascimento, CMC. & Brasil, EUA & DA \\
\hline & 2013. Winchester, J; Gill, DP; Tarnanas, I. & EUA, Grécia & $\begin{array}{l}\text { DA; DA, DL e DV; } \\
\text { DA }\end{array}$ \\
\hline \multirow{4}{*}{ 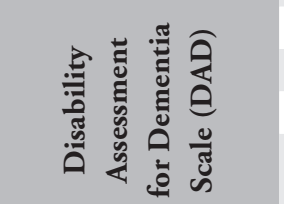 } & 2009. Mioshi, E. & Reino Unido & DFT \\
\hline & 2010. Bahia, VS. & Brasil & DA \\
\hline & 2012. Nadkarni, NK; Jang, J. & Canadá, Austrália & DA; DA \\
\hline & $\begin{array}{l}\text { 2013. Arrighi, HM; Mioshi, E; Benke, T; } \\
\text { Sikkes, SAM. }\end{array}$ & EUA, Reino Unido, Áustria, Holanda & DA; DFT; DA; DA \\
\hline \multirow{4}{*}{ 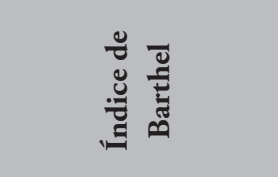 } & 2011. Tanaka, N. & Japão & DA, DV. \\
\hline & 2012. Vreugdenhil, A. & Austrália & $\mathrm{DA} ; \mathrm{DA}$ \\
\hline & 2013. Telenius, EW. & Noruega & DA \\
\hline & 2014. Ha, E. & Coreia & DA \\
\hline \multirow{5}{*}{ 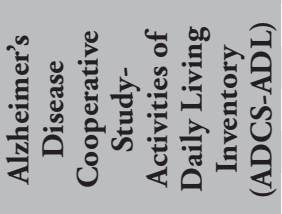 } & 2010. Bhattacharya, S; Rosenthal, E. & Dinamarca, EUA & DA; DDP \\
\hline & 2011. Trigg, R; Ince, A. & Reino Unido, Turquia & $\mathrm{DA}, \mathrm{DA}$ \\
\hline & 2012. Bernick, C. & EUA & DA \\
\hline & 2013. Hallikainen, I. & Finlândia & DA \\
\hline & 2014. Kahle-Wroblesk, K. & EUA & DA \\
\hline
\end{tabular}

${ }^{a}$ Doença de Alzheimer; ${ }^{b}$ Demência Vascular; ' ${ }^{D}$ emência Fronto Temporal; ${ }^{\mathrm{d}}$ Demência da Doença de Parkinson; ${ }^{\mathrm{e}}$ Demência com corpos de Lewy.

A Escala de Atividades Instrumentais de Vida Diária de Lawton e Brody apareceu como o instrumento de avaliação da funcionalidade mais utilizado pelos artigos analisados. Entre os diferentes contextos nos quais a Escala de Atividades Instrumentais de Vida Diária de
Lawton e Brody apareceu, foi mais utilizada quando eram feitas associações e verificação do efeito de fatores comumente avaliados em idosos com demência em relação à funcionalidade, como os sintomas neuropsiquiátricos, a depressão, variáveis clínicas e sobrecarga de 
cuidadores $^{11-23}$. Os demais estudos utilizaram a escala em contextos diversos, que abordavam o efeito de medicamentos na funcionalidade de idosos ${ }^{24}$, a acurácia de escalas de avaliação de AVD em idosos com demência da doença de Parkinson ${ }^{25}$, a validação de outras esca$\operatorname{las}^{26,27}$, a influência do espaço arquitetônico nas $\mathrm{AVD}^{28}$ e, ainda, a capacidade diagnóstica para demências do instrumento em questão ${ }^{29}$.

$\mathrm{O}$ próximo instrumento com maior frequência de utilização foi o Índice de Katz, que foi mais utilizado em estudos que verificaram a influência e relação do declínio da marcha, dos membros superiores e das funçóes executivas sobre a funcionalidade e outras variáveis, como a qualidade de vida, depressão e ansiedade de pacientes com demência ${ }^{11,12,23,30-34}$. Também foi utilizada para validação de novos instrumentos, que auxiliam no cuidado de idosos com demência ${ }^{26,35,36}$. Um estudo fez a relação entre o grau de severidade da demência e o nível de consciência de idosos com o grau funcional dos mesmos ${ }^{20}$.

O Functional Abilities Questionnaire (FAQ), ou Questionário de Atividades Funcionais de Pfeffer, foi o terceiro instrumento mais utilizado. A escala se apresentou como uma medida versátil, pois foi encontrada em diversos contextos nos estudos selecionados. Ela foi utilizada na avaliação da influência do exercício físico no humor, nos sintomas neuropsiquiátricos e nas AVD de idosos com demência ${ }^{12,37-42}$; também na investigação da relação entre função executiva e AVD. O FAQ foi encontrado em estudos que fizeram a diferenciaçấo da taxa de declínio funcional em pacientes com doença de Alzheimer, demência de Lewy e demência vascular ${ }^{43,44}$. Além disso, foi utilizado na validação de dois novos instrumentos e na análise de sua sensibilidade para diferenciar pacientes com Comprometimento Cognitivo Leve daqueles com doença de Alzheimer leve $e^{45-47}$.

O quarto instrumento mais utilizado foi o Disability Assessment for Dementia Scale (DAD), nos estudos, de maneira geral, com o objetivo de avaliar as AVD de idosos portadores de demência frontotemporal, DA e outros tipos ${ }^{48-55}$. Destaca-se também a presença de um estudo que teve como intuito realizar a validação dessa escala para a realidade brasileira ${ }^{(50)}$.

O Îndice de Barthel apareceu como o quinto instrumento mais utilizado pelos estudos encontrados. Os objetivos dos oito estudos que utilizaram o Índice de Barthel estavam direcionados à avaliação da qualidade de vida de idosos com demência, avaliaçáo das AVD na DA e outras demências, utilização desse instrumento para o rastreio de demência e para a validaçáo de outros testes $22,56-62$.

Em relação ao instrumento Alzheimer's Disease Cooperative Study - Activities of Daily Living Inventory (ADCS-ADL), foi identificado como o sexto mais frequente e mais utilizado pelos Estados
Unidos. O ADCS-ADL foi utilizado em diferentes contextos, mas principalmente em estudos que faziam relaçóes entre a demência e a qualidade de vida e que buscavam determinar a influência da idade no curso da $\mathrm{DA}^{63-66}$. Encontrado também em estudos de validação e adequação de escalas ${ }^{67,68}$, esteve presente ainda em um estudo que determinou a estrutura fatorial da ADCS-ADL ${ }^{(69)}$.

Além desses instrumentos mais frequentes, outros também foram encontrados, e mesmo sendo pouco utilizados em estudos, é interessante destacar alguns deles: Activities of Daily Living International Scale (ADL-IS), Bayer - Activities of Daily Living Scale (B-ADL), Blessed Activities of Daily Living Scale, Bristol Activities of Daily Living Scale, Medida de Independência Funcional (MIF), Direct Assessment of Functional Status (DAFS), Activities of Daily Living Questionnaire (ADLQ), Chair Sit to Stand Test, Jebsen Tempo Time (JTT), Timed 8-foot Walk, Yale Physical Activity Survey (YPAS) e Clinical Dementia Rating (CDR $)^{47,59,70-78}$.

É válido destacar, ainda, que a demência mais estudada entre os artigos destacados foi a DA, estando presente em 58 estudos e sendo avaliada com outros tipos de demência ou isoladamente. Logo em seguida vem a DV, que foi identificada sete vezes; DDP, que foi avaliada em seis estudos; DFT, que foi encontrada em cinco; e DL, vista em três estudos.

\section{DISCUSSÃO}

Diante dos resultados apresentados, os instrumentos que apareceram com maior frequência no presente estudo (Lawton and Brody, FAQ, Índice de Katz, DAD, Índice de Barthel e ADCS-ADL) corroboram os instrumentos frequentemente utilizados na avaliaçáo do estado funcional de idosos com demência encontrados em outras revisões sistemáticas recentes. Além disso, os instrumentos que não apareceram com maior frequência e foram apresentados nos resultados, como o ADL-IS, Blessed Activities of Daily Living Scale, Bristol Activities of Daily Living Scale, MIF e DAFS, também estão presentes nas revisóes encontradas ${ }^{8,79-82}$.

Ressalta-se, também, que tanto na presente revisão quanto nas encontradas para comparação dos resultados há instrumentos específicos para a avaliação de idosos com demência, bem como instrumentos que podem ser utilizados em qualquer população de idosos ${ }^{7,81}$.

Além dos instrumentos de avaliação da funcionalidade de idosos com demência relatados acima, essas revisóes sistemáticas identificaram outros que não foram encontrados no presente estudo, como o Cleveland Scale for Activities of Daily Living (CSADL), Interview for Deterioration in Daily 
Living Activities in Dementia (IDDD), Informant Questionnaire on Cognitive Decline in the Elderly (IQCODE), Southampton Assessment of Mobility (SAM) e Teste Funcional ${ }^{79,80,82}$. Essa diferença entre os resultados pode ser elucidada pelo fato da presente revisão ter limitado a procura das escalas para os últimos cinco anos, ao passo que outras revisôes adotaram prazos maiores para a identificação dos instrumentos.

Também é relevante discutir o grande número de estudos e diferentes instrumentos encontrados nessa revisão, pois é possível notar o quão grande é a variedade de escalas e questionários que estão sendo utilizados nas avaliaçốes da funcionalidade de idosos com demência em diversos países e contextos. Dentre eles, é interessante destacar o estudo que utilizou o CDR para a avaliação do comprometimento funcional de idosos com demência ${ }^{76}$. Comumente, esse teste não é utilizado diretamente na avaliação da funcionalidade, mas na identificação e classificação do grau de demência dos indivíduos; porém, como esse questionário possui itens relacionados à capacidade de realização das $\mathrm{AVD}^{83}$, o CDR pode ser uma opçáo viável nesse tipo de avaliação. Esse achado corrobora o estudo de Canon e Novelli ${ }^{82}$, uma revisão sistemática que teve o objetivo de encontrar os instrumentos de avaliação funcional em demência utilizados no Brasil. Como resultado, o estudo identificou também o CDR como um instrumento utilizado para a avaliação da funcionalidade.

Testes como Chair Sit to Stand Test, Jebsen Tempo Time (JTT), Timed 8-foot Walk, Yale Physical Activity Survey $\left(\right.$ YPAS) ${ }^{75}$, encontrados entre os 62 diferentes instrumentos, são escalas que possuem o objetivo de avaliar o desempenho das funçóes motoras e físicas dos indivíduos, mas que nesses determinados estudos foram utilizados diretamente na avaliação da performance funcional. Outros testes, como o Timed Up and Go (TUG), Physical Performance Test (PPT), Sit-toStand test, Trailmaking Test, Digit Symbol, entre outros, que também avaliam as funçóes físicas, motoras e cognitivas, estavam presente em muitos estudos, mas não foram incluídos nos resultados porque avaliavam de forma secundária os aspectos funcionais, sendo que a avaliação primária da funcionalidade era realizada por outros instrumentos, como o FAQ, a Escala de Atividades Instrumentais de Vida Diária de Lawton e Brody e o Índice de Katz.

A presença de estudos que utilizaram testes que avaliam habilidades físicas, motoras e cognitivas vem ao encontro do próprio conceito guarda-chuva de funcionalidade, pois a capacidade do indivíduo em realizar de forma independente suas atividades diárias depende diretamente de seu bom desempenho nas funçôes físicas, motoras e cognitivas.
Diante do que foi encontrado e discutido até o momento, pode-se considerar que os instrumentos que avaliam a funcionalidade são parte importante na avaliação de idosos com demência. Nessa revisão foram destacados os mais utilizados, a fim de elucidar o cenário das pesquisas na área de demências e apresentar como os idosos estão sendo avaliados no que diz respeito à funcionalidade atualmente.

Destaca-se, ainda, a variedade de contextos em que esses instrumentos foram utilizados, pois em nenhum momento foram aplicados de forma independente. Sempre havia outras variáveis de avaliação envolvidas, como a qualidade de vida, desempenho físico e motor, sintomas depressivos e neuropsiquiátricos, sobrecarga dos cuidadores, entre outros, além da presença de outros testes e questionários que avaliavam tais variáveis. Assim, aponta-se, novamente a questáo do quanto o conceito de funcionalidade é amplo e do quanto envolve diversos aspectos da saúde dos indivíduos.

Algumas escalas enfatizadas no estudo foram utilizadas no contexto brasileiro, portanto é importante que se invista em estudos que façam a validação delas. As pesquisas brasileiras dessa área perdem valor e confiabilidade em comparação a estudos internacionais, que em sua maioria utilizam instrumentos validados para a própria realidade.

Como dificuldade do estudo, pode-se apontar o fato de ter sido encontrado um número grande de estudos que utilizavam instrumentos de funcionalidade no contexto da demência. Tornou-se trabalhoso identificar cada um deles, pois foram encontradas diferentes denominaçóes para os mesmos questionários.

\section{CONCLUSÃO}

Após análise dos resultados obtidos na presente revisão, encontrou-se que a Escala de Atividades Instrumentais de Vida Diária de Lawton e Brody, o Índice de Katz, o Questionário de Atividades Funcionais de Pfeffer, o DAD, o Índice de Barthel e o ADCS-ADL são os instrumentos mais utilizados na avaliação da funcionalidade de idosos com demência.

Espera-se que os resultados obtidos nesse estudo possam ilustrar a necessidade de esclarecimento do conceito de funcionalidade e a necessidade da delimitaçáo em questão de número de instrumentos para a avaliação da funcionalidade do idoso com demência. Um aspecto tão importante de uma síndrome tão grave como a demência deve ser padronizado quando for avaliado em futuras pesquisas, principalmente porque as demências são um problema de saúde pública atual e prenunciam continuar sendo por muitos anos. 


\section{AGRADECIMENTOS}

Às alunas da disciplina de Demências: abordagem multidisciplinar e enfoque interdisciplinar de um grave problema de saúde pública do Programa de PósGraduação em Enfermagem da Universidade Federal de São Carlos, que colaboraram com sugestôes (Estela Barbosa, Daniela Pedrazzani, Estefani Rossetti, Ana Cecília Soares, Mariélli Terassi, Érica Nestor, Gabriela Gesualdo e Natália Alves).

Aos autores, que gentilmente cederam seus artigos na íntegra. A Rafael Campanari pela elaboração do software Minilist. À FAPESP pelo auxílio financeiro.

\section{REFERÊNCIAS}

1. Ramos LR. Fatores determinantes do envelhecimento saudável em idosos residentes em centro urbano: Projeto Epidoso, São Paulo. Cad Saude Publica. 2003;19:793-801.

2. Vieira EBKA. Avaliação cognitiva: tratado de geriatria e gerontologia. Rio de Janeiro: Guanabara Koogan; 2002.

3. Njegovan VHM, Mitchell SL, Molnar FJ. The hierarchy of functional loss associated with cognitive decline in older persons. J Gerontol A Biol Sci Med Sci. 2001;56:43-9.

4. Gordilho ASJ, Sérgio J, Silvestre J, Ramos LR, Freire MPA, Espindola N, et al. Desafios a serem enfrentados no terceiro milênio pelo setor saúde na atenção ao idoso. Rio de Janeiro: Universidade Aberta da Terceira Idade; 2000.

5. Bressan LA. O desempenho funcional dos idosos com demência [tese]. Ribeirão Preto: Faculdade de Medicina de Ribeirão Preto, Universidade de São Paulo; 2005.

6. Matsudo SMM. Avaliação do idoso: física e funcional. Londrina: Midiograf; 2000.

7. Fló CM, Perracini MR. Funcionalidade e envelhecimento. In:_ Funcionalidade e envelhecimento. São Paulo: Guanabara Koogan; 2011. p. 3-24.

8. Paixão Jr. CM, Reichenheim ME. Uma revisão sobre instrumentos de avaliaçáo do estado funcional do idoso. Cad Saude Publica. 2005;21:7-19.

9. Moher DLA, Tetzlaff J, Altman DG. Preferred reporting items for systematic reviews and meta-analyses: the PRISMA statement. Ann Intern Med. 2009;151(4):264-70.

10. Ursi E. Prevenção de lesôes de pele no perioperatório: revisão integrativa da literatura [dissertação]. Ribeirão Preto: Universidade de São Paulo; 2005.

11. Monaci L, Morris RG. Neuropsychological screening performance and the association with activities of daily living and instrumental activities of daily living in dementia: baseline and 18- to 24-month follow-up. Int J Geriatr Psychiatry. 2012;27(2):197-204. PubMed PMID: 22223145.

12. Marra TA, Pereira DS, Faria CD, Tirado MG, Pereira LS. Influence of socio-demographic, clinical and functional factors on the severity of dementia. Arch Gerontol
Geriatr Suppl. 2011;53(2):210-5. PubMed PMID: 21109312.

13. Ouchi YAK, Meguro M, Kasai M, Ishii H, Meguro K. Impaired instrumental activities of daily living affect conversion from mild cognitive impairment to dementia: the Osaki-Tajiri Project. Psychogeriatrics. 2012;12:34-42.

14. Rasovska H, Rektorova I. Instrumental activities of daily living in Parkinson's disease dementia as compared with Alzheimer's disease: relationship to motor disability and cognitive deficits: a pilot study. J Neurol Sci. 2011;310(12):279-82. PubMed PMID: 21851954.

15. Tran M, Bedard M, Dubois S, Weaver B, Molloy DW. The influences of psychotic symptoms on the activities of daily living of individuals with Alzheimer disease: a longitudinal analysis. Aging Ment Health. 2013;17(6):738-47. PubMed PMID: 23425341

16. Hesseberg $\mathrm{K}$, Bentzen $\mathrm{H}$, Ranhoff $\mathrm{AH}$, Engedal $\mathrm{K}$, Bergland A. Disability in instrumental activities of daily living in elderly patients with mild cognitive impairment and Alzheimer's disease. Dement Geriatr Cogn Disord. 2013;36(3-4):146-53. PubMed PMID: 23900051.

17. Fukui T, Lee E. Visuospatial function is a significant contributor to functional status in patients with Alzheimer's disease. Am J Alzheimers Dis Other Demen. 2009;24(4):31321. PubMed PMID: 19403740.

18. Balci BD, Yener G, Angin S. The relationship between physical performance, cognition and depression in alzheimer type of dementia. J Neurol Sci. 2011;28(1):051-7.

19. Jonas C, Schiffczyk C, Lahmeyer C, Mueller F, Riepe MW. Staging dementia using proxy-reported activities of daily living. Dement Geriatr Cogn Disord. 2011;32(2):111-7. PubMed PMID: 21952470.

20. Amanzio M, Vase L, Leotta D, Miceli R, Palermo S, Geminiani G. Impaired awareness of deficits in Alzheimer's disease: the role of everyday executive dysfunction. J Int Neuropsychol Soc. 2013;19(1):63-72. PubMed PMID: 22995647.

21. Lucca U, Garri M, Recchia A, Logroscino G, Tiraboschi P, Francheschi M, et al. A population-based study of dementia in the oldest old: the Monzino 80-plus Study. BMC neurol. 2011;11:54.

22. Vreugdenhil A, Cannell J, Davies A, Razay G. A community-based exercise programme to improve functional ability in people with Alzheimer's disease: a randomized controlled trial. Scand j caring sci. 2012;26(1):12-9. PubMed PMID: 21564154.

23. D’Onofrio G, Sancarlo D, Panza F, Copetti M, Cascavilla L, Paris F, et al. Neuropsychiatric symptoms and functional status in Alzheimer's disease and vascular dementia patients. Curr Alzheimer Res. 2012;9(6):759-71. PubMed PMID: 22715983.

24. Wattmo C, Wallin AK, Londos E, Minthon L. Longterm outcome and prediction models of activities of daily living in Alzheimer disease with cholinesterase inhibitor 
treatment. Alzheimer dis assoc disord. 2011;25(1):63-72. PubMed PMID: 20847636.

25. Christ JB, Fruhmann Berger M, Riedl E, Prakash D, Csoti I, Molt W, et al. How precise are activities of daily living scales for the diagnosis of Parkinson's disease dementia? A pilot study. Parkisionism relat disord. 2013;19(3):371-4. PubMed PMID: 23231974.

26. Farina E, Fioravanti R, Pignatti R, Alberoni M, Mantovani F, Manzoni G, et al. Functional living skills assessment: a standardized measure of high-order activities of daily living in patients with dementia. Eur $\mathrm{j}$ phys rehabil med. 2010;46(1):73-80. PubMed PMID: 20332730.

27. Manni R, Sinforiani E, Zucchella C, Terzaghi M, Rezzani C. A sleep continuity scale in Alzheimer's disease: validation and relationship with cognitive and functional deterioration. Neur Sci. 2013;34(5):701-5. PubMed PMID: 22622870.

28. Marquardt G, Johnston D, Black BS, Morrison A, Rosenblatt A, Lyketsos CG, et al. Association of the spatial layout of the home and ADL abilities among older adults with dementia. Am J Alzheimer's Dis Other Demen. 2011;26(1):51-7. PubMed PMID: 21282278.

29. Koskas P, Henry-Feugeas MC, Feugeas JP, Poissonnet A, Pons-Peyneau C, Wolmark Y, et al. The Lawton instrumental activities daily living/activities daily living scales: a sensitive test to Alzheimer disease in community-dwelling elderly people? J Geriatr Psychiatr Neurol. 2014;27(2):8593. PubMed PMID: 24578460.

30. Hebert LE, Bienias JL, McCann JJ, Scherr PA, Wilson RS, Evans DA. Upper and lower extremity motor performance and functional impairment in Alzheimer's disease. Am J Alzheimer's Dis Other Demen. 2010;25(5):425-31. PubMed PMID: 20484749.

31. Paula JJD, Malloy-Diniz LF. Executive functions as predictors of functional performance in mild Alzheimer's dementia and mild cognitive impairment elderly. Estud psicol (Natal). 2013;18:117-24.

32. Rolland Y, Cantet C, Barreto PS, Cesari M, Kan GA, Vellas B. Predictors of decline in walking ability in community-dwelling Alzheimer's disease patients: Results from the 4-years prospective REAL.FR study. Alzheimer's Res Ther. 2013;5:52.

33. Mougias AA, Politis A, Lyketsos CG, Mavreas VG. Quality of life in dementia patients in Athens, Greece: predictive factors and the role of caregiver-related factors. Int Psychogeriatr. 2011;23(3):395-403. PubMed PMID: 20701816.

34. Volkers KM, Scherder EJ. The effect of regular walks on various health aspects in older people with dementia: protocol of a randomized-controlled trial. BMC geriatr. 2011;11:38. PubMed PMID: 21827648. Pubmed Central PMCID: PMC3176485.

35. Correia CC, Lima F, Junqueira F, Campos MS, Bastos O, Petribu K, et al. AD8-Brazil: cross-cultural validation of the ascertaining dementia interview in Portuguese. J Alzheimers Dis. 2011;27(1):177-85. PubMed PMID: 21799253.

36. Lee SB, Park JR, Yoo JH, Park JH, Lee JJ, Yoon JC, et al. Validation of the dementia care assessment packet-instrumental activities of daily living. Psychiatry Investig. 2013;10(3):238-45. PubMed PMID: 24302946. Pubmed Central PMCID: PMC3843015.

37. Winchester J, Dick MB, Gillen D, Reed B, Miller B, Tinklenberg J, et al. Walking stabilizes cognitive functioning in Alzheimer's disease (AD) across one year. Arch gerontol geriatr. 2013;56(1):96-103. PubMed PMID: 22959822. Pubmed Central PMCID: PMC3766353.

38. Clare L, Whitaker CJ, Nelis SM, Martyr A, Markova IS, Roth I, et al. Multidimensional assessment of awareness in early-stage dementia: a cluster analytic approach. Dement geriatr cogn disord. 2011;31(5):317-27. PubMed PMID: 21508642.

39. Brown PJ, Devanand DP, Liu X, Caccappolo E. Functional impairment in elderly patients with mild cognitive impairment and mild Alzheimer disease. Arch gen psychiatr. 2011;68(6):617-26. PubMed PMID: 21646578. Pubmed Central PMCID: PMC3682408.

40. Nascimento CMC, Teixeira CVL, Gobbi LTB, Gobbi S, Stella F. A controlled clinical trial on the effects of exercise on neuropsychiatric disorders and instrumental activities in women with Alzheimer's disease. Braz j phys ther. 2012;16:197-204.

41. Marshall GA, Rentz DM, Frey MT, Locascio JJ, Johnson KA, Sperling RA. Executive function and instrumental activities of daily living in mild cognitive impairment and Alzheimer's disease. Alzheimers Dement. 2011;7(3):300-8. PubMed PMID: 21575871. Pubmed Central PMCID: PMC3096844.

42. Díaz CYE, Socarrás ACR, Torres KB. Deterioro cognitivo, demencia y estado funcional de residentes en un hogar de ancianos: estudio descriptivo de corte transversal con abordaje cuantitativo. Medwave. 2012;12(11).

43. Gill DP, Hubbard RA, Koepsell TD, Borrie MJ, Petrella RJ, Knopman DS, et al. Differences in rate of functional decline across three dementia types. Alzheimers Dement. 2013;9(5 Suppl):S63-71. PubMed PMID: 23643459. Pubmed Central PMCID: PMC3766452.

44. Gill DP, Koepsell TD, Hubbard RA, Kukull WA. Risk of decline in functional activities in dementia with Lewy bodies and Alzheimer disease. Alzheimer disease and associated disorders. 2011;25(1):17-23. PubMed PMID: 21192240. Pubmed Central PMCID: PMC3053143.

45. Bahia VS, Carthery-Goulart MT, Novelli MM, Kato-Narita EM, Areza-Fegyveres R, Caramelli P, et al. Functional disability in Alzheimer disease: a validation study of the Brazilian version of the Disability Assessment for Dementia (DAD-Br). Alzheimer dis assoc disord. 2010;24(3):291-5. PubMed PMID: 20473140.

46. Teng E, Becker BW, Woo E, Knopman DS, Cummings JL, Lu PH. Utility of the functional activities 
questionnaire for distinguishing mild cognitive impairment from very mild Alzheimer disease. Alzheimer dis assoc disord. 2010;24(4):348-53. PubMed PMID: 20592580. Pubmed Central PMCID: PMC2997338.

47. Tarnanas I, Schlee W, Tsolaki M, Müri R, Mosimann U, Nef T. Ecological validity of virtual reality daily living activities screening for early dementia: longitudinal study. JMIR Serious Games. 2013;1.

48. Arrighi HM, Gelinas I, McLaughlin TP, Buchanan J, Gauthier S. Longitudinal changes in functional disability in Alzheimer's disease patients. Int Psychogeriatr. 2013;25(6):929-37. PubMed PMID: 23406898.

49. Mioshi E, Hodges JR, Hornberger M. Neural correlates of activities of daily living in frontotemporal dementia. J geriatr psychiatr neurol. 2013;26(1):51-7. PubMed PMID: 23427102.

50. Mioshi E, Kipps CM, Hodges JR. Activities of daily living in behavioral variant frontotemporal dementia: differences in caregiver and performance-based assessments. Alzheimer dis assoc disord. 2009;23(1):70-6. PubMed PMID: 19266701.

51. Nadkarni NK, Levy-Cooperman N, Black SE. Functional correlates of instrumental activities of daily living in mild Alzheimer's disease. Neurobiol aging. 2012;33(1):53-60. PubMed PMID: 20359778. Pubmed Central PMCID: PMC3897231.

52. Benke T, Delazer M, Sanin G, Schmidt H, Seiler S, Ransmayr G, et al. Cognition, gender, and functional abilities in Alzheimer's disease: how are they related? J alzheimers dis. 2013;35(2):247-52. PubMed PMID: 23388173.

53. Lam LC, Lui VW, Luk DN, Chau R, So C, Poon V, et al. Effectiveness of an individualized functional training program on affective disturbances and functional skills in mild and moderate dementia--a randomized control trial. Int $\mathrm{j}$ geriatr psychiatry. 2010;25(2):133-41. PubMed PMID: 19582757.

54. Sikkes SA, Pijnenburg YA, Knol DL, de Lange-de Klerk ES, Scheltens P, Uitdehaag BM. Assessment of instrumental activities of daily living in dementia: diagnostic value of the Amsterdam Instrumental Activities of Daily Living Questionnaire. J geriatr psychiatr neurol. 2013;26(4):24450. PubMed PMID: 24212244.

55. Jang J, Cushing N, Clemson L, Hodges JR, Mioshi E. Activities of daily living in progressive non-fluent aphasia, logopenic progressive aphasia and Alzheimer's disease. Dement geriatr cogn disord. 2012;33(5):354-60. PubMed PMID: 22796966.

56. Garre-Olmo J, Lopez-Pousa S, Turon-Estrada A, Juvinya D, Ballester D, Vilalta-Franch J. Environmental determinants of quality of life in nursing home residents with severe dementia. J am geriatr soc. 2012 Jul;60(7):1230-6. PubMed PMID: 22702541.

57. Ha E, Kim K. Factors that influence activities of daily living in the elderly with probable dementia. J psychiatr ment health nurs. 2014 Jun;21(5):447-54. PubMed PMID: 24112355.

58. Telenius EW, Engedal K, Bergland A. Physical performance and quality of life of nursing-home residents with mild and moderate dementia. Int $\mathrm{j}$ environ res public health. 2013;10(12):6672-86. PubMed PMID: 24317384. Pubmed Central PMCID: PMC3881134.

59. Tanaka N, Nakatsuka M, Ishii H, Nakayama R, Hosaka R, Meguro K. Clinical utility of the functional independence measure for assessment of patients with Alzheimer's disease and vascular dementia. Psychogeriatrics. 2013;13(4):199205. PubMed PMID: 24289460.

60. Lin LP, Hsu SW, Hsia YC, Wu CL, Chu C, Lin JD. Association of early-onset dementia with activities of daily living (ADL) in middle-aged adults with intellectual disabilities: the caregiver's perspective. Res dev disabil. 2014 Mar;35(3):626-31. PubMed PMID: 24467810.

61. Luttenberger K, Schmiedeberg A, Grassel E. Activities of daily living in dementia: revalidation of the E-ADL Test and suggestions for further development. BMC psychiatry. 2012;12:208. PubMed PMID: 23176536. Pubmed Central PMCID: PMC3605268.

62. Chen CY, Leung KK, Chen CY. A quick dementia screening tool for primary care physicians. Arch gerontol geriatr. 2011;53(1):100-3. PubMed PMID: 20638142.

63. Bhattacharya S, Vogel A, Hansen ML, Waldorff FB, Waldemar G. Generic and disease-specific measures of quality of life in patients with mild Alzheimer's disease. Dement geriatr cogn disord. 2010;30(4):327-33. PubMed PMID: 20924186.

64. Trigg R, Watts S, Jones R, Tod A. Predictors of quality of life ratings from persons with dementia: the role of insight. Int j geriatr psychiatry. 2011;26(1):83-91. PubMed PMID: 21157853.

65. Rosenthal E, Brennan L, Xie S, Hurtig H, Milber J, Weintraub D, et al. Association between cognition and function in patients with Parkinson disease with and without dementia. Mov disord publisher. 2010 Jul 15;25(9):11706. PubMed PMID: 20310053. Pubmed Central PMCID: PMC2963089. Epub 2010/03/24. eng.

66. Bernick C, Cummings J, Raman R, Sun X, Aisen P. Age and rate of cognitive decline in Alzheimer disease: implications for clinical trials. Arch neurol. 2012;69(7):901-5. PubMed PMID: 22431834.

67. Hallikainen I, Hanninen T, Fraunberg M, Hongisto K, Valimaki T, Hiltunen A, et al. Progression of Alzheimer's disease during a three-year follow-up using the CERADNB total score: Kuopio ALSOVA study. Int psychogeriatr. 2013;25(8):1335-44. PubMed PMID: 23676340.

68. Ince A, Mavioğlu H, Eser E. Adaptation to Turkish community and reliability-validity of ADCS-ADL scale. J neurol sci. 2011;28(1):4.

69. Kahle-Wrobleski K, Coley N, Lepage B, Cantet C, Vellas $\mathrm{B}$, Andrieu S, et al. understanding the complexities of 
functional ability in Alzheimer's disease: more than just basic and instrumental factors. Curr Alzheimer Res. 2014;11(4):357-66.

70. Lehfeld H SS, Hoerr R, Stemmler M. SKT Short Cognitive Performance Test and Activities of Daily Living in Dementia. GeroPsych. 2014;27(2):75-80.

71. Umayal S, Kulathunga M, Somaratne S, Srikanth S, Kathriarachchi S, De Silva R. Validation of a functional screening instrument for dementia in an elderly sri lankan population: comparison of modified bristol and blessed activities of daily living scales. BMC res notes. 2010;3:268. PubMed PMID: 20974013. Pubmed Central PMCID: PMC2987868.

72. Talmelli LFdS, Vale FdACd, Gratão ACM, Kusumota L, Rodrigues RAP. Doença de Alzheimer: declínio funcional e estágio da demência. Acta paul enferm. 2013;26:219-25.

73. Gitlin LN, Winter L, Dennis MP, Hodgson N, Hauck WW. A biobehavioral home-based intervention and the well-being of patients with dementia and their caregivers: the COPE randomized trial. JAMA. 2010;304(9):983-91. PubMed PMID: 20810376.

74. Munoz-Neira C, Lopez OL, Riveros R, Nunez-Huasaf J, Flores P, Slachevsky A. The technology - activities of daily living questionnaire: a version with a technology-related subscale. Dement geriatr cogn disord. 2012;33(6):361-71. PubMed PMID: 22797087.

75. Steinberg M, Leoutsakos JM, Podewils LJ, Lyketsos CG. Evaluation of a home-based exercise program in the treatment of Alzheimer's disease: the Maximizing Independence in Dementia (MIND) study. International journal of geriatric psychiatry. 2009;24(7):680-5. PubMed PMID: 19089875.

76. Treiber KA, Carlson MC, Corcoran C, Norton MC, Breitner JC, Piercy KW, et al. Cognitive stimulation and cognitive and functional decline in Alzheimer's disease: the cache county dementia progression study. J Gerontol B Psychol Sci Soc Sci. 2011;66(4):416-25. PubMed PMID: 21441386. Pubmed Central PMCID: PMC3132266.

77. Razani J, Bayan S, Funes C, Mahmoud N, Torrence N, Wong J, et al. Patterns of deficits in daily functioning and cognitive performance of patients with Alzheimer disease. J geriatr psychiatr neurol. 2011;24(1):23-32. PubMed PMID: 21164171.

78. Razani J, Wong JT, Dafaeeboini N, Edwards-Lee T, Lu P, Alessi C, et al. Predicting everyday functional abilities of dementia patients with the Mini-Mental State Examination. J geriatr psychiatr neurol. 2009;22(1):62-PubMed PMID: 19196632. Pubmed Central PMCID: PMC2679691.

79. Sikkes SA, de Lange-de Klerk ES, Pijnenburg YA, Scheltens P, Uitdehaag BM. A systematic review of Instrumental Activities of Daily Living scales in dementia: room for improvement. J neurol, neurosurg psychiatry. 2009;80(1):712. PubMed PMID: 19091706.

80. Chaves ML GC, Porto CS, Mansur L, Carthery-Goulart MT, Yassuda MS, et al. Cognitive, functional and behavioral assessment: Alzheimer's disease. Dement Neuropsychol. 2011;5(3):153-66.

81. Castilla-Rilo J, Lopez-Arrieta J, Bermejo-Pareja F, Ruiz M, Sanchez-Sanchez F, Trincado R. Instrumental activities of daily living in the screening of dementia in population studies: a systematic review and meta-analysis. Int j geriatr psychiatry. 2007;22(9):829-36. PubMed PMID: 17236250.

82. Canon MBF NM. Estudo dos instrumentos de avaliação funcional em demência comumente utilizados no Brasil. Rev Ter Ocup Univ São Paulo. 2012;23:253-62.

83. Maia ALG, Godinho C, Ferreira ED, Almeida V, Schuh A, Kaye J, et al. Aplicação da versão brasileira da escala de avaliação clínica da demência (Clinical Dementia Rating - CDR) em amostras de pacientes com demência. Arq Neuropsiquiatr. 2006;64:485-9.

\section{Como citar este artigo:}

Almeida ML, Leite JC, Avalhais APS, Melo BRS, Thomaz CC, Botan ZRM, Manzini CSS, Vale FAC. Instrumentos na avaliação da funcionalidade de idosos com demência. Rev. Aten. Saúde. 2016;14(48):76-85. 